\title{
On the fractal dimension of orbits compatible with Tsallis statistics.
}

\author{
A. Carati* \\ September 28, 2007
}

\begin{abstract}
In a previous paper (1) it was shown how, for a dynamical system, the probability distribution function of the sojourn-times in phase-space, defined in terms of the dynamical orbits (up to a given observation time), induces unambiguously a statistical ensemble in phase-space. In the present paper it is shown which is the p.d.f. of the sojourn-times corresponding to a Tsallis ensemble (this, by the way, requires the solution of a problem of a general character, disregarded in paper (1)). In particular some qualitative properties, such as the fractal dimension, of the dynamical orbits compatible with the Tsallis ensembles are indicated.
\end{abstract}

keyword Time-averages, non-equilibrium thermodynamics, Tsallis distributions

\section{Introduction}

In the previous paper (1) (see also (2)), a method was introduced to deal with Statistical Thermodynamics if reference is made to dynamics through time-averages. This can be useful for example when one has to deal with metastable states, in which the system remains frozen far from equilibrium for very long times, so that it is not clear what measure in the phase space should be used (see however refs. (3) and the discussion given below). In such cases, apparently, the only statement one can make is that the macroscopic quantities observed are nothing but the time-averages (up to

\footnotetext{
*Università di Milano, Dipartimento di Matematica Via Saldini 50, 20133 Milano (Italy) E-mail: carati@mat.unimi.it
} 
the observation time-scale) of the relevant dynamical variables. This is in agreement with the opinion expressed by most classics. For example, in the discussion following paper (4), one finds the statement: "Such a conception can be understood only if the meaning of probability of a state is made clear. Suppose that the system, left free to evolve, passes through an infinite sequence of states $\mathcal{Z}_{1}, \ldots, \mathcal{Z}_{l}$ in an order whatsoever; to each state there will correspond a certain frequency of occupation. In a sufficiently long interval of time $T$ there will be a subinterval $\tau_{i}$, during which the system is in the state $\mathcal{Z}_{i}$. If $\tau_{i} / T$ tends to a limit when $T$ increases indefinitely, such a limit will measure the probability of the state $\mathcal{Z}_{i}$ and so on. The probability $W$ of a state is thus defined through its relative frequency when the system is left free to evolve for an indefinite time. " The only addition we make here is that there can exist interesting cases in which the relative frequencies settle down, within a finite observation time, to some apparently stationary values, although they may possibly evolve, on much larger time-scales, to the "true" equilibrium values. The suggestion is thus made that the "physical definition of probability" just mentioned should be applied also to metaequilibrium states.

Indeed, if the system is ergodic, the time-averages over infinite times coincide with the Gibbs phase-averages, but there remains open the problem that nothing is known concerning the time-averages for large but finite times. For example, in the literature cases are reported (see (5)) in which the dimensions of the orbits of Hamiltonian system appear to have noninteger values according to numerical estimates, notwithstanding the fact that the dimension of the full orbit (involving all times) is proven (see (6)) to actually be an integer. This fact was explained in the paper (7). There it was shown, for the familiar standard map, that the observed dimension actually depends on the observation time, and that the attainment of the "true value" (two in that case) would require an exceedingly large number of iterations (outside of the computers reach). This, notwithstanding the fact that, the fractal dimension appears to settle down to a definite noninteger value on a finite time-scale. It is clear that on time-scales of the latter type the sojourn-time measure too has to appear very odd, i.e. non absolutely continuous with respect to the Lebesgue one.

In the paper (1) it was shown that the use of time-averages amounts to introducing a measure in phase space, suitably defined by the dynamics of the system. In short (see Section 2 for precise definitions) one has to determine the statistics of the sojourn-times, and then the coarse-grained density of the phase-space measure turns out to be nothing but the (logarithm of the) Laplace transform of the p.d.f. of the sojourn-time of any cell. In particular, the usual Gibbs measure is recovered if the dynamics is very chaotic, i.e. the p.d.f. (of the sojourn-time) is a Poissonian one.

One can imagine that, if one has to deal with a metastable state (i.e. an 
"ergodic behaviour" is granted only on a times-scale much larger than the available one), then the orbits could exhibit some strange features (such as a non integer fractal dimension on the observed time-scale) which may prevent the use of the Gibbs measure. It has been suggested that in some problems where metastable states show up (as in systems of rotators with long-range coupling (8), or in galaxies (9)), one has to replace the Gibbs measure by the Tsallis one (10). In the present paper we show that, if a system has dynamical time-averages compatible with a Tsallis ensemble, then, on a certain time-scale, the orbits have a definite non integer fractal dimension. We also show that the diffusion process of the orbits in phase space is, in some sense, slower than in the full chaotic case corresponding to Gibbs measure.

As a matter of fact, in proving such results, it occurred to the present author to realise that in order to establish a correspondence between dynamics and Tsallis distribution one has to solve an analytical problem that was disregarded in the previous paper (1). This gap is overcome in the present paper. The point is that an exact correspondence between dynamics and Tsallis distribution can be given only for continuous-time dynamical systems and not for mappings. In the case of a mapping, such a correspondence is obtained by introducing a suitable limiting procedure.

The paper is organized as follows. In Section 2 the method to deal with time-averages is recalled. In Section 3 the limiting procedure mentioned above, necessary to deal with mappings, is discussed. In Section 4 the statistics of the sojourn-times is computed in the case of the Tsallis distribution. In Section 5 the qualitative properties of the dynamical orbits compatible with the Tsallis distributions are derived, and some final comments are added in Section 6. Two Appendices complete the paper.

\section{Time-averages}

We recall here, briefly, the method which was introduced in (1) in order to obtain the relevant thermodynamic functions on the basis of dynamics, namely when use is made of time-averages rather than of ensemble averages.

Consider a diffeomorphism $\Phi$ on a phase-space $\mathcal{M}$, and an orbit $x_{n}=$ $\Phi\left(x_{n-1}\right), n=1, \ldots, N$ up to "time" $N$, determined by an initial value $x_{0}$. As a particular case, one can think of the orbits generated by iterations of the time $-\tau$ map induced by the flow of an autonomous Hamiltonian system. The time-average (up to time $N$ ) of a dynamical variable $A(x)$ (a 
real function on $\mathcal{M}$ ) is defined by

$$
\bar{A}\left(x_{0}\right) \stackrel{\text { def }}{=} \frac{1}{N} \sum_{n=1}^{N} A\left(x_{n}\right) .
$$

Such a time-average can also be computed by partitioning the space $\mathcal{M}$ into a large number $K$ of disjoint cells $\mathcal{Z}_{j}$ (such that $\mathcal{M}=\cup \mathcal{Z}_{j}$ ), and reckoning the number of times $n_{j}\left(x_{0}\right)$ the orbit $\left\{x_{n}\right\}$ visits any cell $\mathcal{Z}_{j}$ (so that $n_{j} / N$ is the discrete analogue of the sojourn time, see also (11), pp. 20-21). Indeed one has

$$
\bar{A}\left(x_{0}\right) \simeq \sum_{j=1}^{K} A_{j} \frac{n_{j}}{N},
$$

where $A_{j}$ is the value of $A$ at a chosen point $x \in \mathcal{Z}_{j}$.

If a certain probability distribution is assigned for the initial data $x_{0}$, then $n_{j}$ turns out to be a random variable with a certain cumulative distribution function $F_{j}(n)$, which gives the a priori probability that the cell $\mathcal{Z}_{j}$ be visited a number of times $n_{j} \leq n$ :

$$
p\left(n_{j} \leq n\right)=F_{j}(n) .
$$

In paper (1) the following hypothesis was introduced:

Hypothesis 1 The quantities $n_{j}$ are independent random variables, conditioned by

$$
\sum n_{j}=N .
$$

One can expect that a diffeomorphism $\Phi$ on a large-dimensional phasespace $\mathcal{M}$ should satisfy this hypothesis. In fact, a single cell $\mathcal{Z}_{j}$ will be transformed by $\Phi$ into a set which will intersect, in general, a large number of different cells $\mathcal{Z}_{k}$ (in principle $2^{d}$ of them, if $d$ is the dimension of the phase-space), so that one can hardly expect a correlation between the numbers $n_{j}$ and $n_{k}$. Things are different for low-dimensional maps, even if they are strongly chaotic: in the one-dimensional case, for example, $\Phi\left(\mathcal{Z}_{j}\right)$ will intersect at most two other cells, say $\mathcal{Z}_{k_{1}}$ and $\mathcal{Z}_{k_{2}}$, so that one has $n_{k_{1}}+n_{k_{2}} \geq n_{j}$ and hypothesis 1 is no more fulfilled.

From the fact that the occupation numbers $n_{j}$ are random variables, there follows that the time-average $\bar{A}\left(x_{0}\right)$ too is a random variable. Notice that, being the sum of many random variables, by the law of large numbers with great probability $\bar{A}\left(x_{0}\right)$ will take values close to its expectation (see Appendix 1).

So it is meaningful to consider the expectation of $\bar{A}\left(x_{0}\right)$ as the quantity of interest for Statistical Mechanics. Denoting by $<\cdot>$ the expectation 
with respect to the a priori distribution, the quantity of interest is then $<\bar{A}>$, which takes the form

$$
<\bar{A}>=\frac{1}{N} \sum_{j=1}^{K} A_{j}<n_{j}>.
$$

Actually, in statistical thermodynamics one does not deal directly with the a priori probability, because it is generally assumed that the time-average of the energy of the system has a given value, which should play the role of an independent variable. So we consider the energy of the system, which we denote by $\varepsilon$, and the corresponding time-average $\bar{\varepsilon}=\sum_{j} \varepsilon_{j} n_{j} / N$, and we impose on the numbers $n_{1}, \cdots, n_{K}$ the further condition

$$
\frac{1}{N} \sum_{j=1}^{K} \varepsilon_{j} n_{j}=U=\text { const . }
$$

Thus the quantity of interest is $\langle\bar{A}\rangle_{U}$, the a posteriori expectation of $\bar{A}$ given $U$.

The problem of computing $<\bar{A}\rangle_{U}$ can be reduced to the computation of the "generating function"

$$
Z(A, \mu) \stackrel{\text { def }}{=} \sum_{\left\{n_{j}\right\}}^{\prime} \exp \left(-\mu \sum A_{j} n_{j}\right) P\left(\left\{n_{j}\right\}\right),
$$

through the relation

$$
<\bar{A}>_{U}=-\left.\frac{1}{N} \frac{\partial}{\partial \mu} \log Z(A, \mu)\right|_{\mu=0} .
$$

Here $\sum^{\prime}$ denotes a sum over the possible sequences $\left\{n_{j}\right\}$ constrained by $1 / N \sum n_{j}=N$ and $\sum \varepsilon_{j} n_{j}=U$. It turns out (see reference (1)) that the asymptotic expansion of the generating function $Z(A, \mu)$ is very simply computed in the limit of very "large" systems (the ones of interest for thermodynamics), by using the steepest descent method. If in such an expansion one retains only the leading term, i.e. one neglects the remainder (an explicit expression of which could however be given), one finds that $<\bar{A}>_{U}$ can be computed by making reference to a function $\chi_{j}(z)$, which is the logarithm of the Laplace transform of the cumulative p.d.f. $F_{j}(n)$, namely is defined by

$$
\exp \left(\chi_{j}(z)\right) \stackrel{\text { def }}{=} \int_{\mathbf{R}} e^{-n z} \mathrm{~d} F_{j} .
$$


Indeed one establishes the relation

$$
<\bar{A}>_{U}=-\frac{1}{N} \sum_{j} A_{j} \chi_{j}^{\prime}\left(\frac{\theta \varepsilon_{j}}{N}+\alpha\right) .
$$

where prime denotes derivative, and $\theta$ and $\alpha$ are parameters determined by the equations

$$
\left\{\begin{array}{l}
U=-\frac{1}{N} \sum_{j} \varepsilon_{j} \chi_{j}^{\prime}\left(\frac{\varepsilon_{j} \theta}{N}+\alpha\right) \\
N=-\sum_{j} \chi_{j}^{\prime}\left(\frac{\varepsilon_{j} \theta}{N}+\alpha\right)
\end{array}\right.
$$

In terms of the quantities

$$
\nu_{j} \stackrel{\text { def }}{=}-\chi_{j}^{\prime}\left(\frac{\varepsilon_{j} \theta}{N}+\alpha\right)
$$

the previous relations can be written as

$$
<\bar{A}>_{U}=\frac{1}{N} \sum_{j} A_{j} \nu_{j}, \quad U=\frac{1}{N} \sum_{j} \varepsilon_{j} \nu_{j}, \quad N=\sum_{j} \nu_{j},
$$

and this shows that $\nu_{j}$ can be interpreted as the mean occupation number of cell $\mathcal{Z}_{j}$. The quantities $\nu_{j} / N$ are then the coarse-grained analogues of the density of the standard equilibrium measures.

In particular, if the process of occupation of any cell is a Poisson one, i.e. if the successive visits of a given cell are independent events, then one finds $\chi_{j}=\chi^{\text {Poiss }}$, where

$$
\chi^{\text {Poiss }}(z)=p e^{-z}-p,
$$

with a parameter $p>0$. In such a case one easily shows that the system follows a Gibbs statistics. In fact the mean occupation numbers $\nu_{j}$ are easily calculated from (7), and turn out to be given by

$$
\nu_{j}=N \frac{e^{-\theta \varepsilon_{k} / N}}{Z(\theta)},
$$

where $Z(\theta) \stackrel{\text { def }}{=} e^{\alpha}=\sum_{j} e^{-\theta \varepsilon_{j} / N}$ is the usual partition function, so that relation (6) becomes the usual canonical one, with $\theta / N$ playing the role of inverse temperature (see also (2)).

Instead, the Tsallis $q$-distribution for $q>1$, which is the one considered in this paper, is obtained if the variables $n_{j}$ are distributed in such a way that $\chi_{j}=\chi^{T s}$, where

$$
\chi^{T s}(z)=p\left(1+\frac{z}{\sigma}\right)^{-\sigma}-p
$$


here $p$ and $\sigma$ are positive parameters, moreover $\sigma$ is related to the so-called "entropic index" $q$ by the relation

$$
\sigma=\frac{1}{q-1}
$$

Notice that $\chi^{T s}$ converges to $\chi^{\text {Poiss }}$ for $\sigma \rightarrow+\infty$, i.e. for $q \rightarrow 1^{+}$. The case $q<0$ could also be dealt with taking $\chi^{T s}(z)=-p z^{\sigma}$, with $0<\sigma<1$, but some analytic techniques different from the ones used here are needed. So, the discussion of the latter case is left for a future work. For what concerns the case $0<q<1$, we have no clear ideas at the moment.

While in the papers (1), (2) the attention was put in determining the form of the thermodynamic functions when the functions $\chi_{j}$ are given (in particular for $\chi_{j}=\chi^{T s}$ ), in the present paper we address instead the problem of "going back to the dynamics". Our aim is indeed to determine the statistics of the occupation numbers, i.e. the form of the cumulative p.d.f. $F_{j}(n)$, when $\chi_{j}=\chi^{T s}$ is given.

\section{Continuous-time systems and Tsallis statis- tics}

Our aim is thus to invert the procedure followed in the paper (1), which amounted to determine the ensembles from the dynamical orbits, because we now aim at determining some statistical properties of the dynamics from the ensemble. To this end some preliminary considerations are needed.

In fact one could naively assume that the "characteristic" functions $\chi_{j}(z)$ are given according to the Tsallis ensemble, i.e. by $\chi_{j}=\chi^{T s}$, and then try to determine the corresponding probabilities $p\left(n_{j}=n\right)$ of the occupation numbers $n_{j}$. But this turns out to be impossible, because of a very well known result (see for example the handbook (12), volume 2nd, page 342), namely

Theorem 1 (Laplace transform of integer-valued variables) A complex function $\hat{f}(z)$ is the Laplace transform of an integer-valued, infinitely divisible random variable, if and only if one has

$$
\hat{f}(z)=\exp \left(\alpha\left[g\left(e^{-z}\right)-1\right]\right),
$$

where $\alpha$ is a real parameter, and the function $g(\zeta)$ satisfies

$$
g(0)=1, \quad \frac{\mathrm{d}^{n}}{\mathrm{~d} \zeta^{n}} g(\zeta) \geq 0 \quad \forall 0 \leq \zeta<1 \text { and } \forall n \geq 0 .
$$


One easily checks that $\exp \left(\chi^{T s}(z)\right)$ does not satisfy the hypothesis of Theorem 1, so that it cannot represent the Laplace transform of any p.d.f $F_{j}(n)$. However, even if the Tsallis statistics cannot be applied directly to the variables $n_{j}$, nevertheless it can be applied to suitably defined variables $\tilde{n}_{j}$ which we now introduce.

To this end we first point out that, as shown in Appendix 2, the function $\exp \left(\chi^{T s}(z)\right)$ can be obtained through a limiting procedure on a family of functions $\hat{f}_{\tau}(z)$ satisfying the requirement of Theorem 1. Precisely one has to consider the limit in which the mean occupation number $\left\langle n_{j}\right\rangle$ of each cell diverges. This occurs, for example, when the orbit $\left\{x_{n}\right\}$ of the considered dynamical system are obtained by iterating the time $-\tau$ map induced by the flow of an autonomous Hamiltonian system, i.e. when one sets $x_{n}=x(n \tau)$, if $x(t)$ denotes the orbits of the continuous-time system. It is clear that, if one lets $\tau$ tend to zero and increases the number of iterations $N$ in such a way that the total time $\tau N$ is kept fixed, on the one hand the occupation numbers $n_{j}$ diverge, while on the other the variables $\tilde{n}_{j} \stackrel{\text { def }}{=} \tau n_{j}$ converge to the value of the "time" spent by the orbit in the cell $\mathcal{Z}_{j}$. By the way, the quantities $\tilde{n}_{j}$ coincide with the quantities $\tau_{j}$ appearing in the quotation from paper (4) mentioned in the Introduction.

Note that the expectation $\langle\bar{A}>$ and the conditional expectation $<$ $\bar{A}>_{U}$ have the same form whether they be expressed in terms of the variables $\tilde{n}_{j}$ (see the formulæ given below) or of the variables $n_{j}$ (see the formulæ (2) and (6) ). In fact for the expectation one has

$$
<\bar{A}>=\frac{1}{\tilde{N}} \sum_{j=1}^{K} A_{j}<\tilde{n}_{j}>,
$$

with $\tilde{N}=\tau N$, and for the conditional expectation one has

$$
<\bar{A}>_{U}=\frac{1}{\tilde{N}} \sum_{j=1}^{K} A_{j} \tilde{\nu}_{j},
$$

where $\tilde{\nu}_{j}$ are defined, analogously to (7), by

$$
\tilde{\nu}_{j} \stackrel{\text { def }}{=}-\tilde{\chi}_{j}^{\prime}\left(\left(\frac{\varepsilon_{j} \theta}{\tilde{N}}+\alpha\right)\right.
$$

while $\exp \left(\tilde{\chi}_{j}(z)\right)$ is the Laplace transform of the p.d.f. $\tilde{F}_{j}$ of the random variables $\tilde{n}_{j}$ (for notational simplicity, the index $\tau$ in $\tilde{n}_{j}, \tilde{N}, \tilde{\chi}_{j}$, is $\sup$ pressed).

Now, if we are considering distribution functions such that

$$
\lim _{\tau \rightarrow 0} \tilde{\chi}_{j}(z)=\chi^{T s}(z)
$$


then in the limit $\tau \rightarrow 0$ all quantities converge to the ones computed using the Tsallis distribution. ${ }^{1}$ Appendix 2 is devoted to exhibit a family of functions $\exp (\tilde{\chi}(z))$, depending parametrically on $\tau$, which satisfy the hypothesis of Theorem 1 and tend to $\exp \left(\chi^{T s}(z)\right)$ as $\tau$ tends to zero. It would be of interest to characterize the class of distributions which have that of Tsallis as a limiting one. For the sake of notational simplicity, the tilde sign will be omitted in the rest of the paper, and correspondingly the variables $n_{j}$ have to be thought of as (well approximated by) continuous ones.

\section{The empirical distribution of $n_{j}$ for orbits obeying the Tsallis statistics.}

We now turn to the computation of the probability $p\left(n_{j}=n\right)$ of the occupation numbers $n_{j}$, having assumed that the functions $\chi_{j}(z)$ are given according to the Tsallis ensemble, i.e. with $\chi_{j}=\chi^{T s}$. This will be easily obtained through the preliminary calculation of the corresponding p.d.f. $f_{j}(n)=f^{T s}(n)$, where $f_{j}(n)$ is the probability density corresponding to the cumulative p.d.f. $F_{j}(n)$. The limiting procedure discussed in the previous Section will now be understood.

So we have to determine $f^{T s}(n)$ by inverting relation (5) (with $\mathrm{d} F_{j}=f_{j} \mathrm{~d} n$ ) in the case $\chi_{j}=\chi^{T s}$. The asymptotic behaviour of $f^{T s}(n)$ for large $n$ can be found in (13); instead we concentrate here on the behaviour for small $n$, which is the case relevant for our porpouses (as will be shown in the next Section).

It turns out that $f^{T s}(n)$ doesn't have a closed expression in terms of known functions, except for some special cases. An example will be given later. An expression for $f^{T s}(n)$ can however be given as a series expansion, namely

$$
f^{T s}(n)=e^{-p} \delta(n)+\frac{e^{-p-\sigma n}}{n} \sum_{k \geq 1} \frac{\left(p \sigma^{\sigma} n^{\sigma}\right)^{k}}{k ! \Gamma(k \sigma)}
$$

where $\Gamma(x)$ is the Euler gamma function.

To this end, one makes reference to well known theorems about the shift and the scaling of the Laplace transform, from which one gets

$$
f^{T s}(n)=\sigma e^{-p-\sigma n} g(\sigma n),
$$

\footnotetext{
${ }^{1} \mathrm{By}$ a well known theorem, the convergence of the Laplace transform also implies the convergence of the function $\tilde{F}_{j}$ to the continuous distribution $F^{T s}$ of the "time" spent by the orbit in the cell $\mathcal{Z}_{j}$.
} 


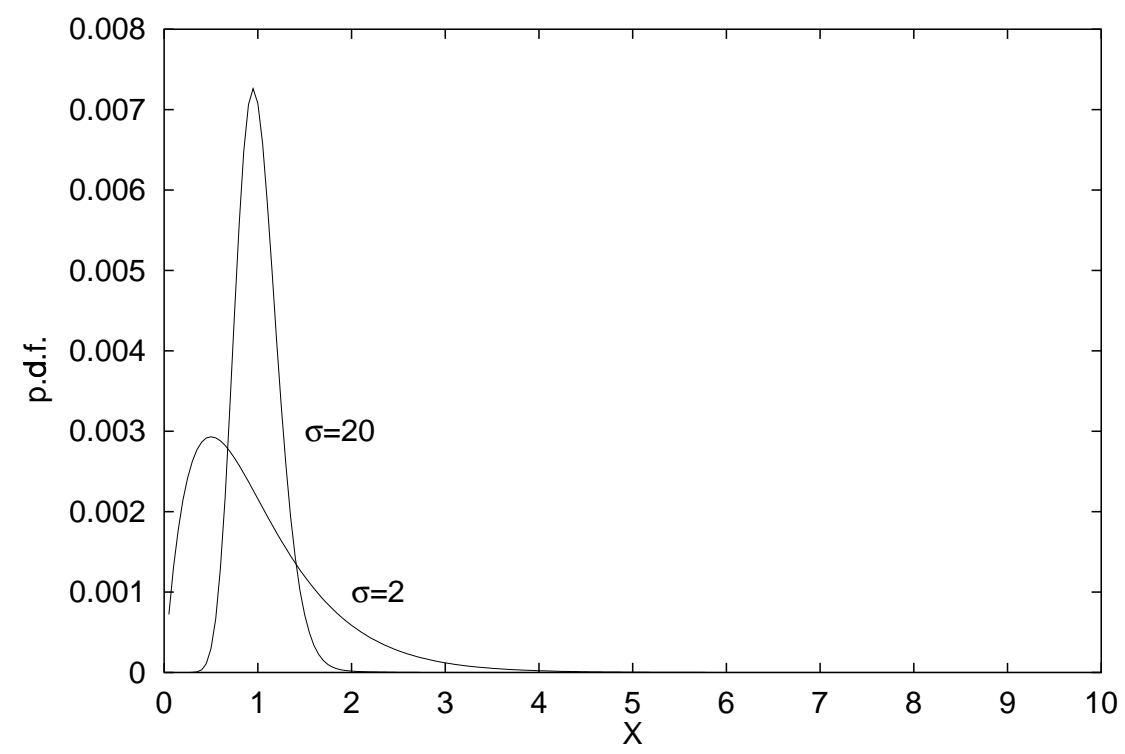

Figure 1: Plot of $f^{T s}(n)$ for $\sigma=2$ and for $\sigma=20$, with $p=0.004$. 
if $g(n)$ satisfies

$$
\int_{0}^{+\infty} \exp (-n z) g(n) \mathrm{d} n=\exp \left(p z^{-\sigma}\right) .
$$

Then, to compute $g(n)$, one considers the series expansion

$$
\exp \left(p z^{-\sigma}\right)=1+\sum_{k \geq 1} \frac{p^{k}}{k ! z^{k \sigma}},
$$

and uses the fact that 1 is the Laplace transform of the Dirac $\delta(n)$, while $1 / z^{k \sigma}$ is the Laplace transform of $n^{k \sigma-1} / \Gamma(k \sigma)$. So one gets

$$
g(n)=\delta(n)+\frac{1}{n} \sum_{k \geq 1} \frac{p^{k} n^{k \sigma}}{k ! \Gamma(k \sigma)},
$$

i.e. (10).

In particular, if one puts $\sigma=1$, the series entering (10) can be explicitly evaluated, and one finds

$$
f^{T s}(n)=e^{-p} \delta(n)+\sqrt{\frac{p}{2 n}} I_{1}(\sqrt{2 p n}) e^{-p-n},
$$

where $I_{1}(x)$ is the modified Bessel function of order 1 . This provides an example in which the Tsallis p.d.f can be expressed in terms of known functions.

The plots of the function $f^{T s}(n)$ given by (10) are reported in Figure 1 for some values of $p$ and $\sigma$. In Figure 2 the same plots are given in semilogarithmic scale in order to appreciate the behaviour for large values of the independent variable.

Remember now that the knowledge of the function $f_{j}(n)$ is not sufficient to determine the probability of the occupation numbers, because the variables $n_{j}$ should satisfy the constraint $\sum n_{j}=N$. One easily shows that, for any p.d.f. $f(n)$, the probability $p\left(n_{j}=n\right)$ is given by

$$
p\left(n_{j}=n\right)=\frac{f(n) e^{-\alpha n}}{\exp (\chi(\alpha))},
$$

with $\alpha$ determined by

$$
\chi^{\prime}(\alpha)=-\frac{N}{K} .
$$

To show this we recall that, according to Hypothesis 1, one has

$$
p\left(n_{j}=n\right)=f\left(n_{j}\right) \cdot p\left(\sum_{i \neq j} n_{i}=N-n_{j}\right),
$$




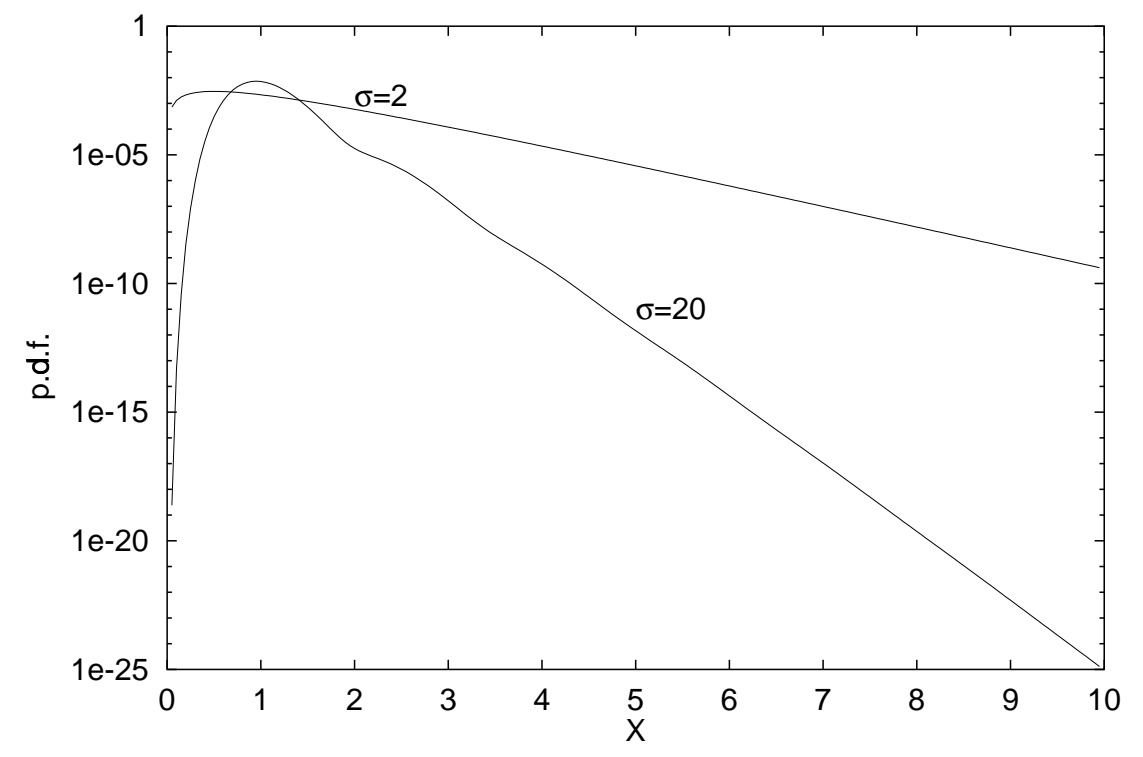

Figure 2: Same as Figure 1, in semi-logarithmic scale. 
so that

$$
p\left(n_{j}=n\right)=\frac{f\left(n_{j}\right) \int^{\prime} \mathrm{d} n_{1} \ldots \mathrm{d} n_{K} \delta\left(\sum_{i \neq j} n_{i}-(N-n)\right) f\left(n_{1}\right) \ldots f\left(n_{K}\right)}{\left.\int \mathrm{d} n_{1} \ldots \mathrm{d} n_{K} \delta\left(\sum_{i} n_{i}-N\right)\right) f\left(n_{1}\right) \ldots f\left(n_{K}\right)},
$$

where $\int^{\prime}$ at the numerator denotes an integration in which the variable $n_{j}$ is excluded. Introducing the representation $\delta(x)=\int \exp (i k x) \mathrm{d} k$ for the Dirac function, one finds

$$
p\left(n_{j}=n\right)=\frac{f\left(n_{j}\right) \int \mathrm{d} k \exp (i k(N-n)+(K-1) \chi(i k))}{\int \mathrm{d} k \exp (i k N+K \chi(i k))} .
$$

Then, using the stationary-phase method one finds

$$
\begin{aligned}
p\left(n_{j}=n\right) & \simeq C f(n) \exp ((K-1) h(N-n / K-1)-K h(N / K)) \\
& \simeq C f(n) \exp \left(-n h^{\prime}(N / K)\right),
\end{aligned}
$$

where $h(\nu)$ is the Legendre transform of $\chi(z)$. Finally, by the Legendre duality one has $h^{\prime}(N / K)=\alpha$, where $\chi^{\prime}(\alpha)=-N / K$, and the constant $C$ is determined by the normalization condition $\int p(n) \mathrm{d} n=1$. This proves formula (11).

\section{The fractal dimensions of the orbits of the Tsallis processes.}

Now, having found the probability distribution (11) for the occupation numbers $n_{j}$, one can compute the mean number $<m>$ of visited cells. This quantity is a very important indicator. As an example, if there exist some constants of motions the mean number of visited cells is expected to be much smaller than for an ergodic system. Likewise, one expects that the mean number of visited cells should scale as some power of $K$ (the total number of cells) rather than as $K$ itself, if the orbits have some fractal structure.

In this connection, one has

Theorem 2 (mean number of visited cells) For the Tsallis distribution, the mean number $\langle m>$ of visited cells is given by

$$
<m>=K \frac{\exp \left((N / K)^{\frac{\sigma}{1+\sigma}} p^{\frac{1}{1+\sigma}}\right)-1}{\exp \left((N / K)^{\frac{\sigma}{1+\sigma}} p^{\frac{1}{1+\sigma}}\right)} .
$$


Proof. This is a simple computation. First, one has that the probability $p(n>0)$ that a cell be visited is given by

$$
p(n>0)=\frac{\int_{n>0} f^{T s}(n) e^{-\alpha n} \mathrm{~d} n}{\exp \left(\chi^{T s}(\alpha)\right)} .
$$

On the other hand, the presence of the term $\delta(n)$ in the expression (10) for $f^{T s}(n)$ implies the the integral $\int_{n>0} p(n) \mathrm{d} n$ can be written as a sum of two terms in the following way

$$
\int_{n \geq 0} \mathrm{~d} n p(n)=\frac{e^{-p}+\int_{n>0} \mathrm{~d} n f^{T s}(n) e^{-\alpha n}}{\exp \left(\chi^{T s}(\alpha)\right)},
$$

so that, from the normalization condition $\int_{n \geq 0} p(n) \mathrm{d} n=1$ one finds

$$
p(n>0)=\frac{\exp \left(\chi^{T s}(\alpha)+p\right)-1}{\exp \left(\chi^{T s}(\alpha)+p\right)} .
$$

Thus, remembering the definition (12) of $\alpha$ and the expression (9) for $\chi^{T s}(z)$, one gets

$$
\frac{\alpha}{\sigma}=-1+\left(\frac{N}{p K}\right)^{\frac{1}{1+\sigma}},
$$

which, together with the relation $<m>=K p(n>0)$, proves relation (13).

Obviously relation (13) shows that the mean number $\langle m\rangle$ of visited cells is equal to $K$ if the argument of the exponentials is large, which typically occurs for large times (i.e. for large $N$ ). Such an expression becomes however particularly meaningful for not too large values of that argument, as occurs in situations in which an orbit starts diffusing into the phase space before covering all the available space.

So, suppose that

$$
\frac{N}{K} \ll \frac{1}{p^{\frac{1}{\sigma}}} ;
$$

in this case one finds that the mean number of visited cells is given by

$$
<m>\simeq N^{\frac{\sigma}{1+\sigma}}(p K)^{\frac{1}{1+\sigma}} .
$$

In the case of a very chaotic motion, one can expect that the number of visited cells increases linearly with time, because the orbit is expected to visit the cells which were not previously visited. This is indeed the case for $\sigma=+\infty$ (corresponding to the Boltzmann-Gibbs case), in which the process is Poissonian. In the Tsallis case, instead, the number of visited 


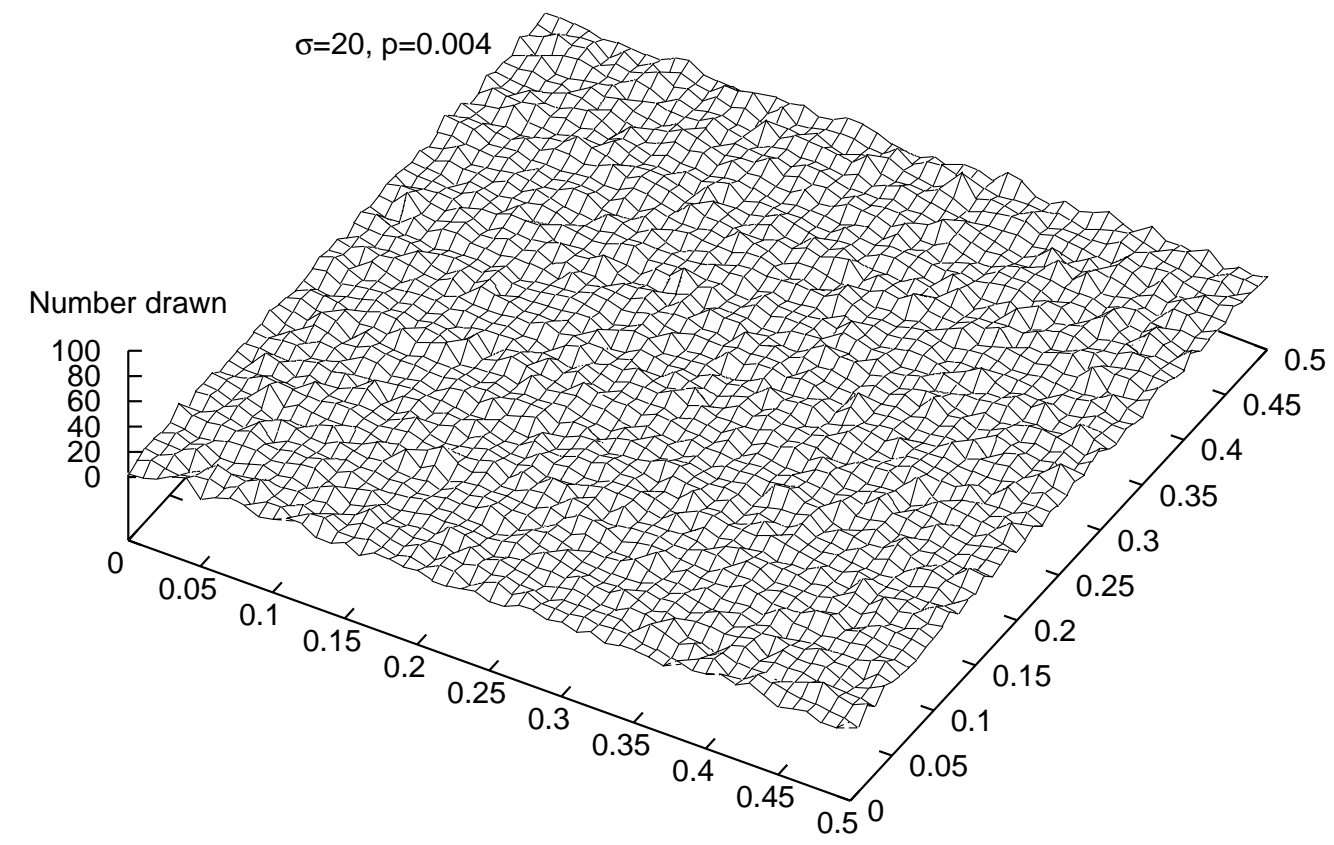

Figure 3: Simulation of a Tsallis dynamics for a map of a square of side 1. The occupation numbers $n_{j}$ of the various cells of the square, i.e. the numbers drawn, are given. Case of a Tsallis distribution near a Poisson one. Notice that only one-fourth of the surface is reported. 
cells increases more slowly (only as $N^{\sigma / 1+\sigma}$ ), i.e. the orbits tend to visit many times the same cell. In fact, the expected number of times $n_{a}$ the orbits visit a given cell (which is surely visited) can be quickly computed by the relation

$$
<m>n_{a}=N
$$

which gives

$$
n_{a}=\left(\frac{N}{p K}\right)^{\frac{1}{1+\sigma}} \gg \frac{N}{K} .
$$

Here the inequality can be easily proved from (15), rising both sides to the power $\sigma /(1+\sigma)$.

We can summarize the previous discussion by saying that for a Tsallis process a small number of cells is visited a large number of times. Thus the sojourn-times distribution tends to become very singular. An illustration of this fact is given in Figures 3 and 4 . We have considered a square of side 1 , divided into $10^{4}$ equal cells (of side $\Delta l=10^{-2}$ ). For each cell $\mathcal{Z}_{j}$ one independently draws a number $n_{j}$ with the probability $p\left(n_{j}=n\right)=$ $f^{T s}(n) e^{-\alpha n} / \exp \left(\chi^{T s}(\alpha)\right)$, i.e. according to the Tsallis distribution, the constant $\alpha$ being determined by the constraint $\sum n_{j}=N=10^{5}$. In short, we simulate a realization of a Tsallis process, which should correspond to a single orbit with initial value taken at random. In the figure we report the surface built by plotting for each cell the drawn number $n_{j}$; remember that $n_{j} / N(\Delta l)^{2}$ gives the density of the sojourn-time measure with respect to the Lebesgue one. In the case of a very chaotic dynamics the density is expected to be smooth, because the fluctuation around the mean value is small for a Poissonian process. In fact Figure 3, which corresponds to the case $p=0.004$ and $\sigma=20$, exhibits a surface considerably smoother than in Figure 4, which refers to the same value of $p$ but to a smaller value of $\sigma$, namely $\sigma=2$ (remember that a high value of $\sigma$ implies that the process is near to a Poisson one). Notice that, in each of the figures, for the sake of clearness only one fourth of the surface is reported.

Another very interesting result is obtained if the value $K$ of cells is increased, as $N$ increases, in such a way as to keep the ratio $N / K$ constant. This corresponds to using a finer partition as the number of iterations is increased. In this case one has the problem of the values to be given to the parameter $p$ when $K$ is changed. For the sake of consistency ${ }^{2}$ one has to

\footnotetext{
${ }^{2}$ In fact, by splitting a cell into two cells, i.e. taking $\mathcal{Z}_{j}=\mathcal{Z}_{j_{1}} \cup \mathcal{Z}_{j_{2}}$, one has $n_{j}=n_{j_{1}}+n_{j_{2}}$. Thus, having assumed that the occupation numbers are independent, one has

$$
\chi_{j}(z)=\chi_{j_{1}}(z)+\chi_{j_{2}}(z)
$$

In terms of the parameter $p$ entering $\chi^{T s}$ through (9), this amounts to the relation $p=2 p^{\prime}$, where $p$ refers to $\chi_{j}$ and $p^{\prime}$ to both $\chi_{j_{1}}$ and $\chi_{j_{2}}$. So, by doubling the number of cells the parameter $p$ is halved.
} 


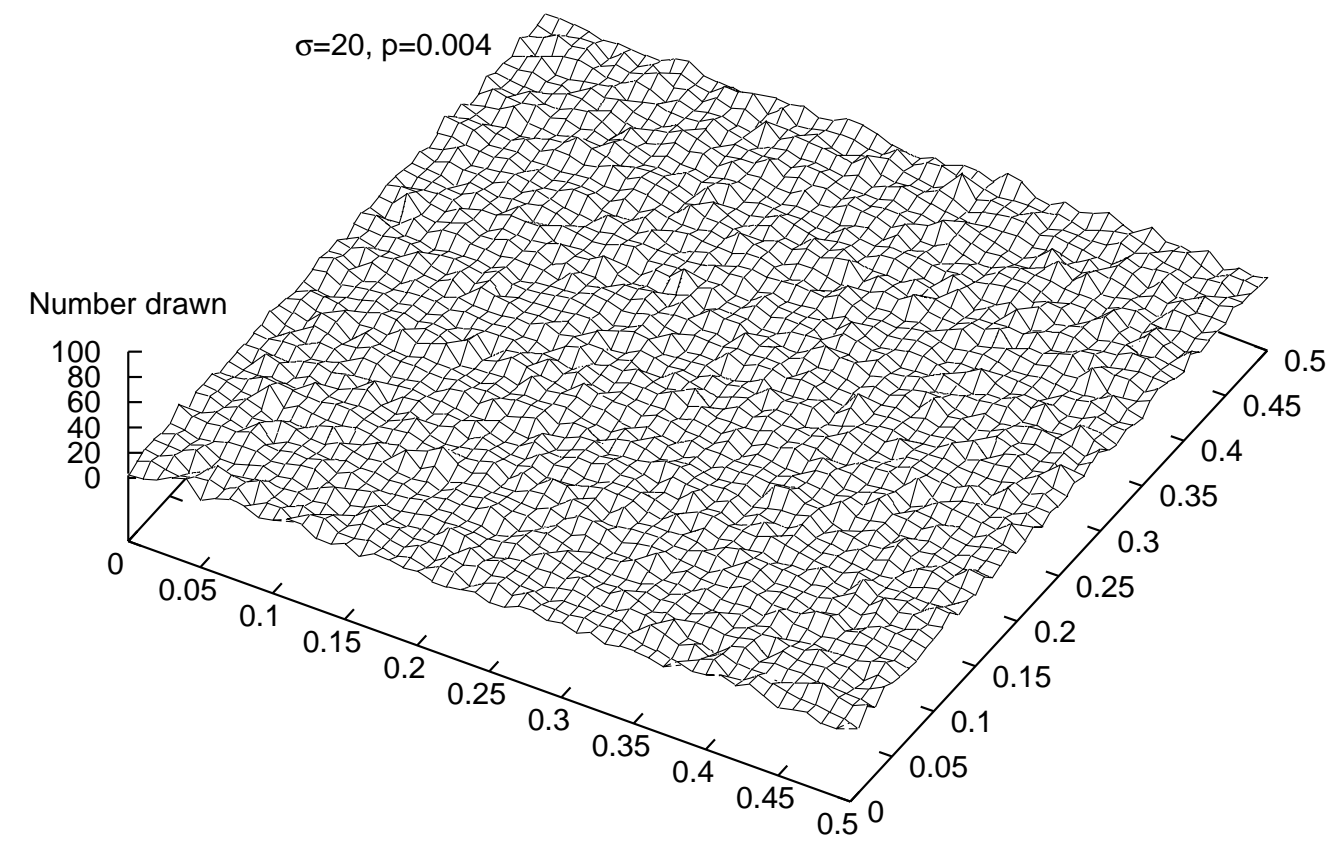

Figure 4: Same as Figure 3 for a Tsallis distribution far from a Poisson one. 
take $p$ proportional to the inverse of the number $K$ of cells, i.e. $p \simeq 1 / K$, so that one finds

$$
\begin{aligned}
<m> & \simeq K^{\frac{\sigma}{1+\sigma}}, \\
n_{a} & \simeq K^{\frac{1}{1+\sigma}} .
\end{aligned}
$$

So, if one uses the box-counting method, the orbit appears as if it had a dimension smaller than that of the phase-space, precisely it appears to have a fractal dimension $d_{f}$ given by

$$
d_{f}=\frac{\sigma}{1+\sigma} d,
$$

where $d$ is the total dimension of the phase-space. Notice that

$$
\frac{\sigma}{1+\sigma}=\frac{1}{q},
$$

where $q$ is the entropic index, so that the latter can be linked to the fractal dimension of the orbit by the relation

$$
d_{f}=\frac{1}{q} d .
$$

Some comments to this relation are deferred to the final Section.

In terms of the sojourn-time measure, one obtains that such a measure is not absolutely continuous with respect to the Lebesgue one, having a support with a dimension smaller than that of the phase-space. This shows that, in order to apply the Tsallis statistics, the orbits must have characteristics very different from those corresponding to uniform chaoticity (as in Anosov systems) usually considered in Statistical Mechanics.

\section{Final comments.}

We have shown how it is possible to determine some properties of the orbits which are compatible with the Tsallis ensemble. Particularly interesting in this connection is, in our opinion, formula (18), namely $d_{f}=d / q$, which relates the entropic index $q$ of the Tsallis distribution to the fractal dimension $d_{f}$ of the corresponding orbits ( $d$ being the dimension of the phase-space). Relations of such a type may be expected, because it is true that, in a sense, the very problem of the existence of entropies different from the Boltzmann-Gibbs-Shannon one did originate in the context of the fractal geometry (see for example (14)). However, to the author's knowledge, in the literature one can find only a few papers devoted to a precise statement 
on the relation between entropic index and fractal dimension. In particular, the papers (15) seem to adopt a point of view close to ours, although obtaining a relation different from ours, because they give $d_{f}=q d$ instead of $d_{f}=d / q$. In this connection one can remark that, as in this paper one deals with the case $q>1$, the formula $d_{f}=q d$ gives a fractal dimension larger than the total one, while our formula (18) gives, coherently, a fractal dimension smaller than the total one. We plan to clarify in a future work the relation between our paper and the just mentioned ones.

There are other papers in the literature which consider the relation between entropic index $q$ and fractal dimension, but they deal just with low-dimensional mappings, to which our method hardly applies. In any case, the comparison with their results seems to be non trivial.

An interesting problem is the physical meaning of the relation (18). Maybe this simply implies that the system behaves as if it had a smaller number of degrees of freedom $(d / q$ instead of $d)$, the other one remaining frozen. The important point is that a fractal structure of the orbits seems to imply that the thermodynamical functions of the system (entropy, free energy, etc.) are different from the ones computed using the Gibbs statistics.

A final comment of a general character concerns the "right" measure to be used in presence of metastable states. In the literature (see for example the papers (3)) one finds the prescription that one should use the Gibbs measure restricted to the phase-space region in which the metastable state is localized. At first sight this seems to be in contrast with the prescription of using the Tsallis measure on the whole phase-space. However, in the end it may turn out the two approaches are not so different, if the domain $D$ in which the metastable state is localized presents a fractal structure. Indeed it can then happen that, at least for some macroscopic observables, the mean value computed with the Gibbs measure restricted to $D$ coincide with the mean value computed in the whole phase-space with a different smooth measure (for example the Tsallis one). This is in fact what has been shown in the present paper, namely that computing averages with respect to the Tsallis measure is equivalent to computing averages in a fractal region carrying a constant measure. The latter measure is actually nothing but the quantity $n_{a} / N$, with $n_{a}$ given in formula (17).

\section{Appendix 1}

We want to show that the time-average $\bar{A}$, as defined by (1), has a variance which is small, and that correspondingly its p.d.f is peaked about its mean. In fact, the variance $\delta_{n_{j}}^{2}$ of the random variable $n_{j}$ can be computed from 
the expression (11) for its p.d.f., and one finds

$$
\delta_{n_{j}}^{2}=\chi_{j}^{\prime \prime}(\alpha) .
$$

In the case of Tsallis, recalling the expressions (9) for $\chi^{T s}(z)$ and (14) for $\alpha$, one gets

$$
\delta_{n_{j}}^{2}=\frac{1+\sigma}{\sigma} \frac{N}{K}\left(\frac{N}{p K}\right)^{1 / 1+\sigma} .
$$

Then, the variance $\delta_{\bar{A}}^{2}$ of the time-average $\bar{A}$, is simply computed by adding the variances of each $n_{j}$, and one finds

$$
\begin{aligned}
\delta_{\bar{A}}^{2} & =\frac{1+\sigma}{\sigma} \frac{1}{(p K)^{1 / 1+\sigma} N^{\sigma / 1+\sigma}} \frac{1}{K} \sum \bar{A}_{j}^{2} \\
& \simeq \frac{1+\sigma}{\sigma} \frac{1}{(p K)^{1 / 1+\sigma} N^{\sigma / 1+\sigma}} \int \bar{A}^{2} \mathrm{~d} \mu .
\end{aligned}
$$

Now, as previously recalled, one has to keep $p$ proportional to the inverse of $K$, so that $p K$ has to be considered essentially constant. So, if $\left(\int \bar{A}^{2} \mathrm{~d} \mu\right)^{1 / 2}$ is of the same order of magnitude as $\langle\bar{A}\rangle$, one gets

$$
\delta_{\bar{A}} \simeq \frac{1}{N^{\sigma / 1+\sigma}}<\bar{A}>,
$$

which implies that, as $N$ grows large, the deviation becomes negligible. This in turn implies that $\bar{A}$ takes values very close to $\langle\bar{A}\rangle$, almost surely.

\section{Appendix 2}

We want to show that there exists a family of p.d.f.'s $F_{\tau}(n)$ concentrated at $\tau n, n=0,1, \cdots$, which converges, as $\tau \rightarrow 0$, to the Tsallis distribution. Consider the function $g(\zeta)$ defined by

$$
g(\zeta)=(a-b \zeta)^{-\sigma},
$$

with $a, b \in \mathbf{R}, a>b>0$. One has

$$
\frac{\mathrm{d}^{n}}{\mathrm{~d} \zeta^{n}} g(z)=\sigma(1+\sigma) \cdots(n-1+\sigma) b^{n}(a-b \zeta)^{-\sigma-n}>0,
$$

for all $0<\zeta<1$ and for $n \geq 0$. Then the function

$$
\hat{f}(z)=\exp \left(\left(a-b e^{-z}\right)^{-\sigma}-(a-b)^{-\sigma}\right),
$$

satisfies the hypothesis of Theorem 1, and so is the Laplace transform of a p.d.f. $F(n)$ concentrated on the integers, for every choice of $a$ and $b$. Then 
the p.d.f. $F_{\tau}(n) \stackrel{\text { def }}{=} F(\tau n)$ will be concentrated at $\tau n, n=0,1, \cdots$, and its Laplace transform will be given by

$$
\begin{aligned}
\hat{f}_{\tau}(z) & =\exp \left(\left(a-b e^{-\tau z}\right)^{-\sigma}-(a-b)^{-\sigma}\right)= \\
& =\exp \left((a-b)^{-\sigma}\left(1+\frac{b\left(1-e^{-\tau z}\right)}{a-b}\right)^{-\sigma}-(a-b)^{-\sigma}\right) .
\end{aligned}
$$

Now, if one chooses $a$ and $b$ as functions of $\tau$ in such a way that $b /(a-b)=$ $1 / q \tau$, while the difference $a-b \stackrel{\text { def }}{=} p$ is independent of $\tau$, i.e. if one sets

$$
a=\frac{p^{-1 / \sigma}(1+\sigma \tau)}{\sigma \tau}, \quad b=\frac{p^{-1 / \sigma}}{\sigma \tau},
$$

one finds

$$
\hat{f}_{\tau}(z)=\exp \left(p\left(1+\frac{\left(1-e^{-\tau z}\right)}{\sigma \tau}\right)^{-\sigma}-p\right) .
$$

Then, letting $\tau \rightarrow 0$, one gets

$$
\hat{f}_{\tau}(z) \rightarrow \exp \left(p\left(1+\frac{z}{\sigma}\right)^{-\sigma}-p\right)=\exp \left(\chi^{T s}(z)\right) .
$$

In such a way, if $n$ is a random variable with p.d.f. $F(n)$, then $\tilde{n}=\lim \tau n$ will be distributed according to Tsallis.

\section{References}

[1] A. Carati, Physica A 348, 110-120 (2005).

[2] A. Carati, Physica A 369, 417-431 (2006).

[3] O. Penrose, J.L. Lebowitz, J. Stat. Phys. 3, 211 (1971);

L.S. Schulman, J. Phys. A: Math. Gen. 13, 237-250 (1980);

S. Goldstein, J.L. Lebowitz, Physica D 193, 53-66 (2004);

F. Leyvraz, H. Larralde, D.P. Sanders, Eur. Phys. J. B 50, 57-61 (2006).

[4] A. Einstein, Contribution to the 1911 Solvey Conference, in The collected papers of Einstein, Princeton U.P. (Princeton, 1993), Vol 3, n. 26.

[5] M. Pettini, A. Vulpiani, Phys. Lett. A 106, 207 (1984).

[6] S.E. Newhouse, Soc. Math. France, Astérisque 51, 223 (1978).

[7] G. Benettin, D. Casati, L. Galgani, A. Giorgilli, L. Sironi, Phys. Lett. A 118, 325 (1986). 
[8] V. Latora, A. Rapisarda, C. Tsallis, Physica A 305129 (2002);

V. Latora, A. Rapisarda, C. Tsallis, Phys. Rev. E 64056134 (2001).

[9] A.R. Plastino, A. Plastino Phys. Lett. A 174, 384 (1993);

J.J. Aly, J. Perez, Phys. Rev. E 60, 5185 (1999);

T. Kronberger, M.P. Leubner, E. van Kampen, Astronomy\&Astrophysics 453, 21-25(2006).

[10] C. Tsallis, J. Stat. Phys. 52, 479 (1988).

[11] C. Beck, F. Schloegl, Thermodynamics of Chaotic Systems, Cambridge University Press (Cambridge, 1993).

[12] W. Feller, An introduction to probability theory and its applications, John Wiley \& Sons (New York, 1968).

[13] S. Abe, Y. Nakada, Physical Review E 74, 021120 (2006).

[14] A. Renyi, Acta Math. Hung. 10, 193-215 (1959);

A. Renyi, in Proceedings of the 4th Berkeley Symposium on Mathematical Statistics and Probability, Vol 1, pp. 547-561, J. Neyman ed., University of California Press (Berkeley, 1961).

[15] C. Tsallis, Fractals 3, 541-547 (1995);

Q.A. Wang, L. Nivanen, A. Le Méhauté, M. Pezeril, Physica A $\mathbf{3 4 0}$ 117-125 (2004). 